Civilian Radioactive Waste Management System Management and Operating Contractor

\title{
Principal Isotope Selection Report
}

\author{
Revision 00 \\ Document Identifier No.: B00000000-01717-5705-00104 REV 00
}

August 28, 1998

Prepared for:

U.S. Department of Energy

Yucca Mountain Site Characterization Project Office

P.O. Box 30307

Las Vegas, NV 89036-0307

Prepared by:

Civilian Radioactive Waste Management System

Management and Operating Contractor

1261 Town Center Drive

Las Vegas, NV 89134

Under Contract Number:

DE-AC08-91RW00134 
Civilian Radioactive Waste Management System

Management and Operating Contractor

\section{Principal Isotope Selection Report}

Document Identifier No.: B00000000-01717-5705-00104 REV 00

August 28, 1998

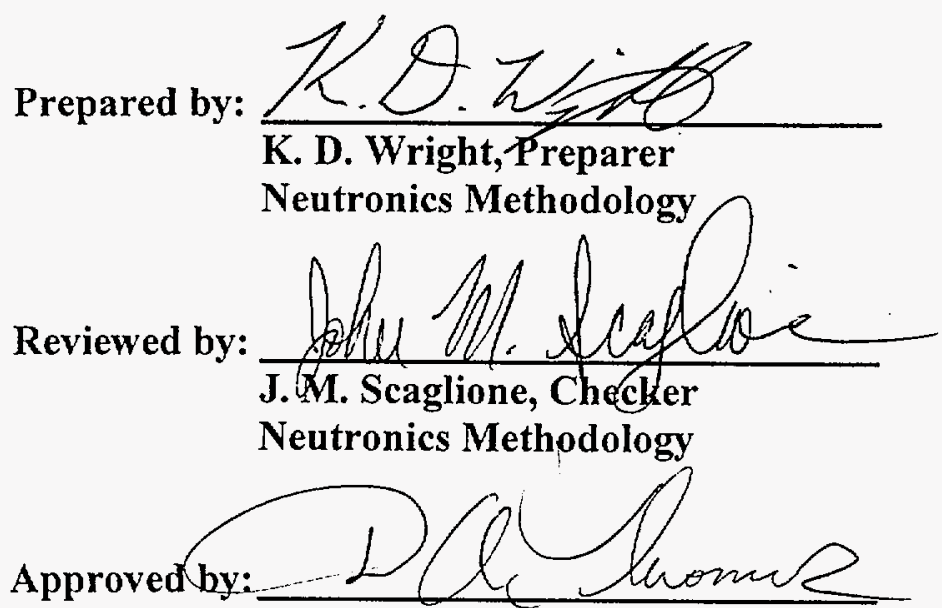

D. A. Thomas, Supervisor

Neutronics Methodology

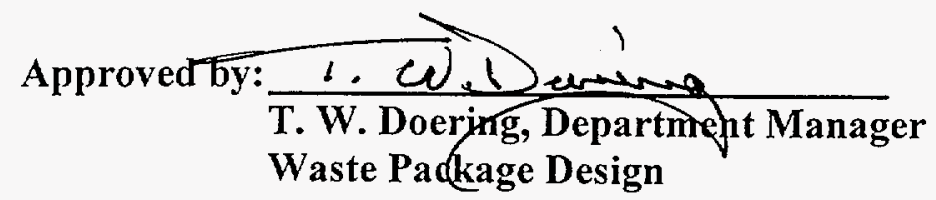

Date: $8 / 28 / 98$

Date: $8 / 28 / 98$

Date: $08 / 28 / 98$

Date: $8 \cdot 28 \cdot 98$ 


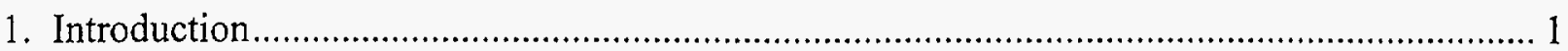

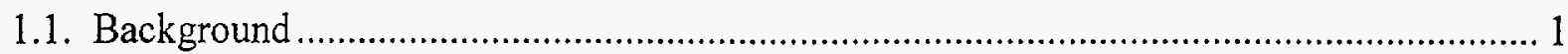

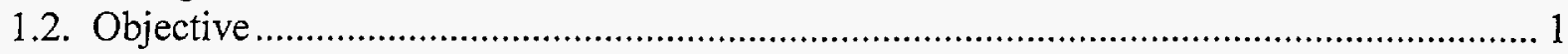

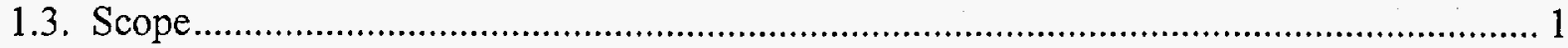

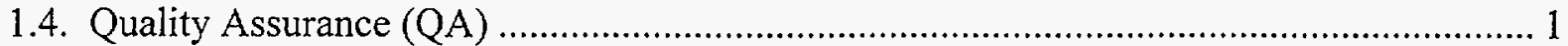

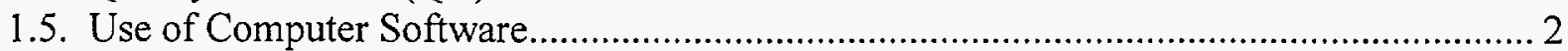

2. Description of the Principal Isotope Set Selection Process ................................................. 2

2.1 Simulation of Hypothetically Degraded SNF ............................................................ 2

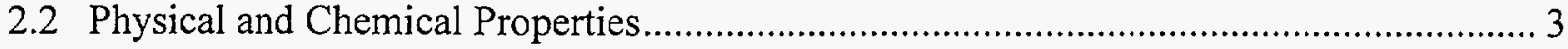

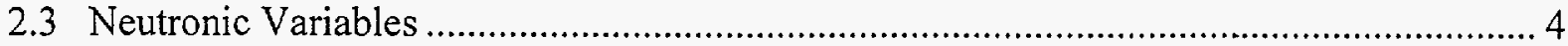

3. Description of Calculations Performed to Support the Selection of Principal Isotopes ........... 5

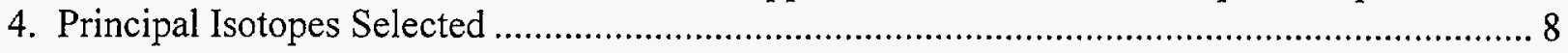

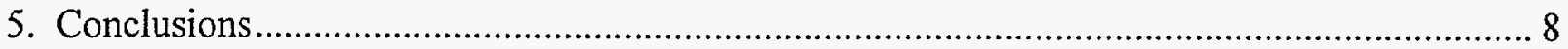

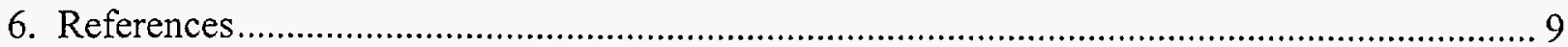

Table

\section{List of Tables}

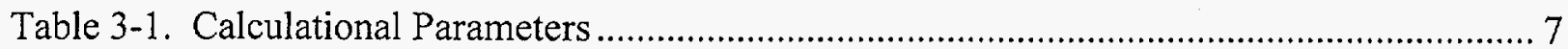

Table 4-1. Selected Principal Isotope Set .................................................................... 8 


\section{Introduction}

Utilizing nuclear fuel to produce power in commercial reactors results in the production of hundreds of fission product and trans-uranic isotopes in the spent nuclear fuel (SNF). When the SNF is disposed of in a repository, the criticality analyses could consider all of the isotopes, some principal isotopes affecting criticality, or none of the isotopes, other than the initial loading. The selected set of principal isotopes will be the ones used in criticality analyses of the SNF to evaluate the reactivity of the fuel/waste package composition and configuration. This technical document discusses the process used to select the principal isotopes and the possible affect that these isotopes could have on criticality in the SNF.

\subsection{Background}

SNF contains hundreds of fission products and trans-uranic actinides. An important design objective for the repository is to ensure that the SNF meets guidelines to maintain a sufficient subcritical margin. Since the economics of commercial reactor operation require the nuclear fuel to be highly burned, it is apparent that the SNF will be highly subcritical. Simulations of critical conditions to evaluate the potential for nuclear criticality could be based on the isotopic concentrations being representative of fresh fuel. While this assumption simplifies nuclear criticality evaluations, it could result in significant economic penalties. Therefore, criticality analyses of SNF, that will be deposited in a repository, will include the effects of burnup on the isotopic concentrations of the fuel.

\subsection{Objective}

The objective of this technical document is to discuss the process used to select the principal isotopes for disposal criticality evaluations with commercial SNF. The principal isotopes will be used as supporting information in the Disposal Criticality Analysis Methodology Topical Report which will be presented to the United States Nuclear Regulatory Commission (NRC) when approved by the United States Department of Energy (DOE) Office of Civilian Radioactive Waste Management (OCRWM).

\subsection{Scope}

This technical document discusses the process for selecting the principal isotopes and the selected principal isotopes to be used in the disposal criticality methodology for SNF. The principal isotopes will be used in supporting documents and as supporting information in the Disposal Criticality Analysis Methodology Topical Report.

\subsection{Quality Assurance (QA)}

The QA program applies to the development of this technical document. The information provided in the technical document is to be indirectly used in the evaluation of the Monitored Geologic Repository waste package and engineered barrier segment. The waste package and engineered barrier segment have been identified as items important to safety, waste isolation, and physical protection of materials in the QAP-2-3 evaluation entitled "Classification of the 
Preliminary MGDS Repository Design" (Ref. 6.1, TBV-228). The Waste Package Design Department (WPDD) responsible manager has evaluated the technical document development activity in accordance with QAP-2-0, "Conduct of Activities." The QAP-2-0 activity evaluation, "Develop Technical Documents" (Ref. 6.2), has determined that the preparation and review of this technical document is subject to "Quality Assurance Requirements and Description" (Ref. 6.3) requirements. As specified in NLP-3-18, "Documentation of QA Controls on Drawings, Specifications, Design Analyses, and Technical Documents," this activity is subject to QA controls.

\subsection{Use of Computer Software}

There was no computer software used in the development of this technical document.

\section{Description of the Principal Isotope Set Selection Process}

The hundreds of fission products and trans-uranic actinides in the SNF significantly reduce the reactivity of the fuel. The repository will be a permanent installation that will contain the fuel material for thousands of years. Since degradation in any hypothetical environment over a period of tens of thousands of years is possible, this degradation must be considered for criticality evaluations. The simulation of hypothetical degradation of the waste package structure and the contained fuel for criticality analyses introduces two of the most important considerations for the selection of a set of principal isotopes to represent the SNF:

1. The simulation of the composition and configuration of the fuel and waste package must include the hypothetical degradation into the most reactive conditions.

2. The selection of the principal isotopes must include consideration of the physicalchemistry properties of the SNF that are consistent with the hypothetical degradation, in addition to the neutronic properties.

Experience with reactor fuel designs and previous preliminary sensitivity evaluations have shown that degraded fuel, within degraded waste packages, can be made to be very reactive. The degraded fuel reactivity can be increased towards a maximum value by increasing the ratio of water moderating material relative to the SNF material.

\subsection{Simulation of Hypothetically Degraded SNF}

There are two important neutronic characteristics of the reactor fuel that influence the selection process for the principal isotopes affecting repository criticality evaluations. The first is the production of isotopes during reactor operation that results in highly reactive SNF when it is discharged. The second is the potential for a highly reactive fuel configuration due to a hypothetically degraded repository/waste package configuration. The first characteristic is discussed in Section 2.3. This section discusses the simulation of the fuel configuration that has been modified to achieve a maximum reactivity in a hypothetically degraded condition. 
With the repository being a permanent installation for the SNF, there are several states of hypothetical degradation. The use of the principal isotopes in criticality evaluations is limited by a degraded state. This limiting degraded state is defined by the cladding material being intact but having some holes that would allow water to be outside of the pellet material, between the pellet and cladding. This limiting degraded state does not consider the pellet material to be mixed with or in solution with the water. Further description of the degraded SNF configuration is provided in Section 3.

Reference 6.4 includes discussions of the neutronic evaluations of the degraded fuel. An important consideration made in the evaluations was that the selection process will be based on the neutronic characteristics of a highly thermalized system. The water moderation is a dominating effect that reduces the importance of fast fissions, increases the resonance escape probability, reduces the neutron leakage and the importance of the water reflection, and enhances the overall effects of thermal reactions on the system's reactivity.

\subsection{Physical and Chemical Properties}

The hypothetical degradation of the fuel and waste package considered in the principal isotope selection evaluation results in holes in the waste package and holes in the fuel cladding. The holes provide the means of allowing water to permeate the SNF environment. The holes also provide the means of allowing the fission products and trans-uranic actinides, that are associated with the pellet-clad gap region, to be displaced.

The displacement of any isotope due to the physical-chemical properties of the respective elements would be extremely difficult to treat in the criticality evaluations. Consequently, the selection process for the principal isotopes is limited by the physical-chemical properties of the elements.

Mendeleev's periodic table of the elements (pp. 16,17, Ref. 6.5) indicates which elements have similar chemical properties and would be unaffected by the fuel degradation, and which elements should not be considered in principal isotope selection. Those elements that would be most affected by the degradation are the inert gases, the halogens, and the alkali metals. These elements were dropped from the principal isotope selection process. Those elements that would be unaffected are the ones with the same principal oxidation state as the actinides. This includes the Lanthanides and the other elements in groups IB through VIIB and VIII.

The isotopes selected as the principal ones are noted in Section 4. These isotopes include 15 fission products and 14 actinides. The selected actinides are stable with respect to their physicalchemical properties in the degraded SNF environment. Of the 15 fission products, 10 are in the group of Lanthanides. This group includes 2 isotopes of Neodynium, 5 of Samarium, 2 of Europium and 1 of Gadolinium. Another 4 of the fission product isotopes, Molybdenum, Technetium, Ruthenium and Rhodium are associated with the stable metals Chromium, Manganese, Iron and Cobalt, respectively. The last of the 15 fission product isotopes is contained in the noble metal group of elements (Silver). The groups of elements representing the fission products are stable with respect to their physical-chemical properties in the degraded SNF environment. 


\subsection{Neutronic Variables}

The hundreds of fission product and trans-uranic isotopes that are in the SNF from commercial power reactors significantly decrease the reactivity of the fuel in comparison to the initial fresh fuel loadings. The criticality evaluations of the repository will insure that there is an appropriate margin of subcriticality for the SNF. Insuring an appropriate subcritical margin for the SNF and, including the reactivity uncertainties associated with the hundreds of fission product and transuranic isotopes introduces too much uncertainty into the criticality evaluations. To reduce the uncertainty, such that a subcritical margin can be evaluated with a sufficiently high degree of confidence, requires that the hundreds of fission product and trans-uranic isotopes be reduced to a set of principal isotopes. Criticality evaluations with the principal isotopes must be based on a high degree of confidence that the predicted concentrations are well known, with a defined uncertainty. In addition, the neutronic properties of the isotopes should include the majority of the neutron productions and absorptions that are associated with the hundreds of fission products and actinides.

The selection process for the principal isotopes was based on identifying the nuclear properties necessary for the isotopes to adequately represent the SNF. Since the isotopes in the SNF are radioactive, they are continually changing with time. Therefore, the time-dependent behavior of the neutron production and absorption must be evaluated. Reference 6.4 contains calculations of the degraded SNF decaying from a period of 5 years through 250,000 years. The results of the calculations indicated that the decay initially causes the reactivity of the SNF to decrease (p. 9, Ref. 6.4). However, after a period of 150 years, the decay caused the SNF reactivity to increase (p. 9, Ref. 6.4). The reactivity peaked at a period of 14,000 years for the most reactive SNF (p. 10, Ref. 6.4).

For other SNF, that is highly reactive, but with lower enrichments than the most reactive fuel, the peak occurred before 14,000 years (p. 10, Ref. 6.4). Following the peak in reactivity, the continuing decay of the SNF resulted in a continuing decrease in reactivity (p. 10, Ref. 6.4). Therefore, the selection process for the principal isotopes was focused on the reactivity of the SNF as a function of time. During the periods of high reactivity at 5 years and 14,000 years (or before) the principal isotopes must have a relatively constant effect on reactivity. Reviewing the selected set of isotopes in Section 4 indicates that these isotopes are generally either stable with no decay or decay with a half-life on the order of several hundred thousand years. Those isotopes that decay in shorter periods have a complementary long half-life isotope that provides a near constant reactivity effect (e.g., the stable isotope Eu-151 is complementary to the short-lived isotope $\mathrm{Sm}-151$ ).

The selection process included a verification that the principal isotopes held a nearly constant reactivity by maintaining a nearly constant absorption fraction, relative to the SNF, with all fission products and actinides included. As discussed in Reference 6.4, the verification included comparisons to different fuel types as well as different operating conditions. Independent of the neutronic variables associated with the fuel types and operating conditions, the selected set of principal isotopes was shown to have at least 96 percent of the absorption as compared to all of the fission products and actinides for the more reactive degraded SNF during the period between the initial decay at 5 years and the peak reactivity around 14,000 years (p. 52, Ref. 6.4). 
Following the peak reactivity period, the less reactive degraded SNF that was highly burned and decayed was shown to have at least 92 percent of the absorption compared to all of the fission products and actinides (p. 52, Ref. 6.4).

\section{Description of Calculations Performed to Support the Selection of Principal Isotopes}

The discussion presented in this section is a summary of the calculations documented in Reference 6.4. The methods used in the calculations to support the selection of the principal isotopes were those of nuclear physics for critical systems, and systems with time dependent isotopics. These methods included neutron transport theory and the theory of nuclear reactions and disintegrations. Neutron transport theory was represented by the time-independent transport equation with a critical eigenvalue representing an asymptotic time period and a buckling eigenvalue representing spatial leakage. The nuclear reactions and disintegrations were represented by a set of coupled differential equations. The solution of the differential equations produced a set of coupled exponential equations (the Bateman equations). These exponential equations represented the time-dependent changes in the isotopics with each product isotope dependent on its own reactions and disintegrations, and on the reactions and disintegrations of the parent and daughter isotopes.

The methods of determining solutions for the isotopic reactions and reactivity effects of the isotopes in the transport equation were based on the XSDRNPM-S computer code module of the SAS2H sequence in the SCALE 4.3 computer software package (p. 6, Ref. 6.4). The XSDRNPM-S results were utilized by the ORIGEN-S computer code (p. 6, Ref. 6.4). The ORIGEN-S module of the SAS2H sequence in the SCALE 4.3 software package used the (Bateman) set of coupled exponential equations to determine the time dependent isotopics within the SNF. The ORIGEN-S results included the reaction rate density along with the isotopic concentration density. The reaction rates were primarily determined in XSDRNPM-S, and collapsed through the COUPLE code module of the SAS2H sequence. The ORIGEN-S edit provided a relative ranking of the isotopic absorption reaction rate density. This ranking provided the means of identifying the principal fission product isotopes affecting critical conditions. This ranking also provided the means of identifying the principal trans-uranic and actinide isotopes affecting critical conditions. The importance of the trans-uranic and actinide isotopes was evaluated using both the relative ranking of the absorption rate and the product of the relative absorption rate and the isotopic $k_{\infty}$. The isotopic $k_{\infty}$ was calculated using the following expression:

$$
k_{\infty}=\left(\frac{\sum_{f_{i}} v_{i}}{\sum_{a_{i}}}\right)=\left(\frac{N_{i} \sigma_{f_{i}} \nu_{i}}{N_{i} \sigma_{a_{i}}}\right)=\left(\frac{\sigma_{f_{i}} v_{i}}{\sigma_{a_{i}}}\right)
$$

where the values of $\sigma_{f_{i}}, v_{i}$, and $\sigma_{a}$, represented the microscopic fission cross section of isotope $i$, the average number of neutrons resulting from thermal neutron induced fission of isotope $i$, and the microscopic absorption cross section of isotope i, respectively (p. 4, Ref. 6.4). The isotopic $k_{\infty}$ was determined with 2200 meter per second $\sigma_{f_{i}}, v_{i}$, and $\sigma_{a_{i}}$ values (p. 4, Ref. 6.4). 
The calculations were performed to provide results that would assist in selecting the principal isotopes which have the largest influence on the SNF reactivity. Therefore, the calculations were performed on a highly reactive degraded SNF configuration. The degraded fuel configuration represented a geometrical arrangement of fuel and moderator which enhanced neutron moderation and thermal neutron interaction within the fuel. Hence, the thermal neutron induced fission rate in the SNF was enhanced resulting in a highly reactive SNF configuration. The degraded fuel configuration was represented as having no grid or other structural material within the assembly lattice, and no material outside the assembly lattice other than a neighboring assembly or a moderator reflector of water. In the degraded configuration, the fuel clad was the only structure and the cladding was degraded to the extent that water was between the pellet and clad. Also in the degraded configuration, the fuel rods and assemblies were further represented as having bowed to the maximum extent within the area of two assembly pitch gap widths. Additionally, the degraded fuel configuration had the following characteristics: (a) no poisons or other elements in the water, (b) water and all material temperatures were decreased to $20^{\circ} \mathrm{C}$, and (c) the water density was maximized at 1.0 gram per cubic centimeter. The lack of soluble poisons, the lack of moderator exclusion, the low temperature conditions, and the maximum hydrogen density all served to maximize the neutron thermalization, thus increasing the thermal neutron population available to induce fission in the SNF.

Since the degraded fuel configuration included degradation of the fuel rod cladding such that water had penetrated the cladding and filled the gap between the fuel pellet and clad, the following two neutron spectrum effects were generated:

1. The material between fuel pellets in the radial plane was greater than before, thereby making each pellet more isolated. The additional material reduced the Dancoff factor.

2. The additional water contributed to a more thermalized neutron spectrum.

Concerning the Dancoff factor, the relative decrease in resonance absorption did not affect the relative absorption rates of the various isotopes with the exceptions of Uranium-238 and Plutonium-239. This was due to the fact that the resonance absorption in the various isotopes other than Uranium-238 and Plutonium-239 was not significant with respect to relative thermal neutron absorption in a configuration characterized by a highly thermalized neutron spectrum.

The calculations used to support the principal isotopes selection process included (a) 18 SAS2H depletion cases (each containing a final depletion pass which represented the SNF at the end of its reactor life in a degraded fuel configuration) and (b) 18 ORIGEN-S decay cases (one case immediately following each of the $18 \mathrm{SAS} 2 \mathrm{H}$ cases to calculate the decay of the SNF to a number of time periods). Table 3-1 lists the parameters that characterized each of the 18 different SAS2H fuel assembly depletion calculations. The infinite neutron multiplication factor $\left(\mathrm{k}_{\mathrm{inf}}\right)$ and relative isotopic absorption rate results, obtained from SAS2H/ORIGEN-S for each of the degraded SNF configurations at 31 different decay times, were used to rank the isotopes according to their effect on SNF reactivity to support selection of the principal isotope set. 
The B\&W 15 by 15 fuel assembly specifications corresponded to the Fuel Batch 3 specifications from the Crystal River Unit 3 reactor (p. 7, Ref. 6.4). The Westinghouse 17 by 17 OFA specifications correspond to the Fuel Batch $6 \mathrm{~B}$ specifications from the McGuire Unit 1 reactor (p. 7, Ref. 6.4). The Westinghouse 17 by 17 standard lattice fuel assembly specifications corresponded to the Fuel Batch 2 specifications from the McGuire Unit 1 reactor (p. 7, Ref. 6.4).

Table 3-1. Calculational Parameters (pp. 7, 8, Ref. 6.4)

\begin{tabular}{|c|c|c|c|c|c|}
\hline $\begin{array}{c}\text { SAS2H/ } \\
\text { ORIGEN-S } \\
\text { Case } \\
\text { Number }\end{array}$ & Fuel Type & $\begin{array}{c}\text { Enrichment } \\
(\mathrm{wt} \% \mathrm{U}-235 \text { in } \\
\left.\mathrm{U} \text { of } \mathrm{UO}_{2}\right)^{3}\end{array}$ & $\begin{array}{c}\text { Burnup } \\
(\mathbf{M W d} / \mathbf{M T U})^{1}\end{array}$ & Assembly Power & Control Rods \\
\hline 1 & $\begin{array}{c}\text { B\&W } \\
15 \text { by } 15\end{array}$ & 2.5 & 18,000 & $\begin{array}{c}100 \% \\
14.5085 \mathrm{MW}^{2}\end{array}$ & None \\
\hline 2 & $\begin{array}{c}\text { B\&W } \\
15 \text { by } 15\end{array}$ & 2.5 & 30,000 & $\begin{array}{c}100 \% \\
14.5085 \mathrm{MW} \\
\end{array}$ & None \\
\hline 3 & $\begin{array}{c}\text { B\&W } \\
15 \text { by } 15\end{array}$ & 5.0 & 30,000 & $\begin{array}{c}50 \% \\
7.2542 \mathrm{MW}\end{array}$ & None \\
\hline 4. & $\begin{array}{c}\text { B\&W } \\
15 \text { by } 15\end{array}$ & 5.0 & 30,000 & $\begin{array}{c}100 \% \\
14.5085 \mathrm{MW}\end{array}$ & None \\
\hline 5 & $\begin{array}{c}\text { B\&W } \\
15 \text { by } 15\end{array}$ & 5.0 & 30,000 & $\begin{array}{c}100 \% \\
14.5085 \mathrm{MW} \\
\end{array}$ & Inserted \\
\hline 6 & $\begin{array}{c}\text { B\&W } \\
15 \text { by } 15\end{array}$ & 5.0 & 63,000 & $\begin{array}{c}100 \% \\
14.5085 \mathrm{MW}\end{array}$ & None \\
\hline 7 & $\begin{array}{l}\text { Westinghouse } \\
\text { OFA } 17 \text { by } 17\end{array}$ & 2.5 & 18,000 & $\begin{array}{c}100 \% \\
17.6736 \mathrm{MW}\end{array}$ & None \\
\hline 8 & $\begin{array}{l}\text { Westinghouse } \\
\text { OFA } 17 \text { by } 17\end{array}$ & 2.5 & 30,000 & $\begin{array}{c}100 \% \\
17.6736 \mathrm{MW}\end{array}$ & None \\
\hline 9 & $\begin{array}{l}\text { Westinghouse } \\
\text { OFA } 17 \text { by } 17\end{array}$ & 5.0 & 30,000 & $\begin{array}{c}50 \% \\
8.8368 \mathrm{MW}\end{array}$ & None \\
\hline 10 & $\begin{array}{l}\text { Westinghouse } \\
\text { OFA } 17 \text { by } 17\end{array}$ & 5.0 & 30,000 & $\begin{array}{c}100 \% \\
17.6736 \mathrm{MW} \\
\end{array}$ & None \\
\hline 11 & $\begin{array}{l}\text { Westinghouse } \\
\text { OFA } 17 \text { by } 17\end{array}$ & 5.0 & 30,000 & $\begin{array}{c}100 \% \\
17.6736 \mathrm{MW}\end{array}$ & Inserted \\
\hline 12 & $\begin{array}{l}\text { Westinghouse } \\
\text { OFA } 17 \text { by } 17\end{array}$ & 5.0 & 63,000 & $\begin{array}{c}100 \% \\
17.6736 \mathrm{MW}\end{array}$ & None \\
\hline 13 & $\begin{array}{l}\text { Westinghouse } \\
\text { Std. } 17 \text { by } 17\end{array}$ & 2.5 & 18,000 & $\begin{array}{c}100 \% \\
17.6736 \mathrm{MW} \\
\end{array}$ & None \\
\hline 14 & $\begin{array}{l}\text { Westinghouse } \\
\text { Std. } 17 \text { by } 17\end{array}$ & 2.5 & 30,000 & $\begin{array}{c}100 \% \\
17.6736 \mathrm{MW}\end{array}$ & None \\
\hline 15 & $\begin{array}{l}\text { Westinghouse } \\
\text { Std. } 17 \text { by } 17\end{array}$ & 5.0 & 30,000 & $\begin{array}{c}50 \% \\
8.8368 \mathrm{MW}\end{array}$ & None \\
\hline 16 & $\begin{array}{l}\text { Westinghouse } \\
\text { Std. } 17 \text { by } 17\end{array}$ & 5.0 & 30,000 & $\begin{array}{c}100 \% \\
17.6736 \mathrm{MW} \\
\end{array}$ & None \\
\hline 17 & $\begin{array}{l}\text { Westinghouse } \\
\text { Std. } 17 \text { by } 17\end{array}$ & 5.0 & 30,000 & $\begin{array}{c}100 \% \\
17.6736 \mathrm{MW}\end{array}$ & Inserted \\
\hline
\end{tabular}


Table 3-1. Calculational Parameters (pp. 7, 8, Ref. 6.4)

\begin{tabular}{|c|c|c|c|c|c|}
\hline $\begin{array}{l}\text { SAS2H/ } \\
\text { ORIGEN-S } \\
\text { Case } \\
\text { Number }\end{array}$ & Fuel Type & $\begin{array}{c}\text { Enrichment } \\
(w \mathrm{w} \% \mathrm{U}-235 \text { in } \\
\left.\mathrm{U} \text { of } \mathrm{UO}_{2}\right)^{\mathbf{3}}\end{array}$ & $\begin{array}{c}\text { Burnup } \\
(\mathrm{MWd} / \mathrm{MTU})^{1}\end{array}$ & Assembly Power & Control Rods \\
\hline 18 & $\begin{array}{l}\text { Westinghouse } \\
\text { Std. } 17 \text { by } 17\end{array}$ & 5.0 & 63,000 & $\begin{array}{c}100 \% \\
17.6736 \mathrm{MW} \\
\end{array}$ & None \\
\hline
\end{tabular}

${ }^{1} \mathrm{MWd} / \mathrm{MTU}=$ megawatt days per tonne of Uranium

${ }^{2} \mathrm{MW}=$ megawatt

${ }^{3} \mathrm{wt} \%=$ weight percent

\section{Principal Isotopes Selected}

The selected principle isotope set consists of 14 actinides and 15 fission products. Table 4-1 presents the selected principle isotope set.

Table 4-1. Selected Principal Isotope Set

\begin{tabular}{|c|c|c|c|c|}
\hline \multicolumn{5}{|c|}{ Actinides } \\
\hline U-233 & U-234 & U-235 & U-236 & U-238 \\
\hline Np-237 & Pu-238 & Pu-239 & Pu-240 & Pu-241 \\
\hline Pu-242 & Am-241 & Am-242m & Am-243 & \\
\hline \multicolumn{5}{|c|}{ Fission Products } \\
\hline Mo-95 & Tc-99 & Ru-101 & Rh-103 & Ag-109 \\
\hline Nd-143 & Nd-145 & Sm-147 & Sm-149 & Sm-150 \\
\hline Sm-151 & Sm-152 & Eu-151 & Eu-153 & Gd-155 \\
\hline
\end{tabular}

\section{Conclusions}

The set of 29 principal isotopes shown in Table 4-1 were selected based on the results of the calculations and evaluations documented in Reference 6.4 and described in Sections 2 and 3 of this document. The 29 principal isotopes contain 14 actinide and 15 fission product isotopes. The neutronic properties of the principal isotopes should include the majority of the neutron productions and absorptions that are associated with the hundreds of fission products and actinides that are actually in the SNF. Independent of fuel type and operational history, the selected 29 principal isotopes account for at least 96 percent of the total neutron absorption in the more reactive degraded SNF configuration, and at least 92 percent of the total neutron absorption in the less reactive (highly burned) degraded SNF configuration (p. 52, Ref. 6.4). The actinides in the selected principal isotope set represent approximately 99 percent of the total neutron production in the degraded SNF configuration (p. 52, Ref. 6.4).

The selected set of 29 principal isotopes is acceptable for quality affecting activities and for use in analyses affecting procurement, construction, or fabrication. The classification for the repository (which includes the waste package) carries TBV-228 because of the preliminary status of the basis for the Monitored Geologic Repository design. This technical document 
conservatively assumes that the resolution of TBV -228 will find the waste package to be quality affecting; consequently, use of any of the data reported herein does not need to carry TBV-228.

\section{References}

6.1. Classification of the Preliminary MGDS Repository Design. Document Identifier Number (DI\#): B00000000-01717-0200-00134 REV 00, Civilian Radioactive Waste Management System (CRWMS) Management and Operating Contractor (M\&O).

MOL.19980211.1192.

6.2. QAP-2-0 Activity Evaluation. Activity Evaluation Number: WP-06, Develop Technical Documents, CRWMS M\&O, August 3, 1997. MOL.19971215.0080.

6.3. Quality Assurance Requirements and Description. DOE/RW-0333P, REV 8, United States Department of Energy Office of Civilian Radioactive Waste Management. MOL.19980601.0022.

6.4. Principal Isotope Selection Calculation. DI\#: B00000000-01717-0210-00031 REV 00, CRWMS M\&O. MOL.19980806.0608.

6.5. Nuclides and Isotopes, Chart of the Nuclides, Fourteenth Edition. General Electric Company, 1989. TIC\#: 201637. 